\title{
LIFTING FORMULAS II
}

\author{
BORIS SHOIKHET
}

\section{Introduction}

Let $\mathfrak{A}$ be an associative algebra, we will also think of $\mathfrak{A}$ as a Lie algebra with the bracket $[a, b]=a \cdot b-b \cdot a$. Suppose that there is a trace $\operatorname{Tr}: \mathfrak{A} \rightarrow \mathbb{C}$ on the associative algebra $\mathfrak{A}$ (this means that $\operatorname{Tr}([A, B])=0$ for any $A, B \in \mathfrak{A}$ ) and a set of its (exterior) derivations $\left\{D_{1}, \ldots, D_{n}\right\}$; such that the following conditions 1) -2$)$ hold:

1) $\operatorname{Tr}\left(D_{i} A\right)=0$ for all $i=1, \ldots, n$ and all $A \in \mathfrak{A}$;

2) $\left[D_{i}, D_{j}\right]=0$ for all $i, j$.

It was proven in [Sh] (and probably elsewhere as well) that if conditions 1) and 2) hold, then

$$
\Psi_{n+1}\left(A_{1}, \ldots, A_{n+1}\right)=\operatorname{Alt}_{A} \underset{D}{\operatorname{Alt}} \operatorname{Tr}\left(D_{1} A_{1} \cdot \ldots \cdot D_{n} A_{n} \cdot A_{n+1}\right)
$$

is a $(n+1)$-cocycle on the Lie algebra $\mathfrak{A}$.

Furthermore, let us suppose that instead of condition 2) we have the weaker condition (i) + (ii):

(i) $\left[D_{i}, D_{j}\right]=\operatorname{ad} Q_{i j}$ for all $i, j=1, \ldots, n \quad\left(Q_{i j} \in \mathfrak{A}\right.$ and $\left.Q_{i j}=-Q_{j i}\right)$

(ii) $\underset{i, j, k}{\operatorname{Alt}} D_{k}\left(Q_{i j}\right)=0$ for all $i, j, k$.

Condition (ii) is analogous to the Jacobi identity. In fact, one can deduce from the Jacobi identity that $\operatorname{Alt}_{i, j, k} D_{k}\left(Q_{i j}\right)$ lies in the center of the Lie algebra $\mathfrak{A}$.

When conditions 1), (i) and (ii) hold, a formula for the $(n+1)$-cocycle on the Lie algebra $\mathfrak{A}$ was found in [Sh]; this formula is quantization on $Q_{i j}$ of the formula (1). This quantization is called the lifting formula. The fact that the lifting formula in reality defines a cocycle was the Main Conjecture in [Sh].

The present paper contains the solution of the following two problems:

First Problem 1. When conditions 1$)-2)$ above hold $\left(\left[D_{i}, D_{j}\right]=0\right)$, to construct formulas for $n+3, n+5, n+7, \ldots$ - cocycles, which are analogous to the formula (1).

Second Problem 1. To construct a quantization of these formulas on $Q_{i j}$, when condition 2 ) is replaced by the condition $(i)+(i i)$.

Received February 26, 1998. 
The First Problem is solved completely in Section 1; the answer to the Second Problem is given in Theorem 2.3; we prove this Theorem as well as the Main Conjecture from [Sh] in Section 3.

Conditions $(1)+(i)+(i i)$ arise in the following situation. Let $\mathfrak{A}=\Psi \operatorname{Dif}_{n}\left(S^{1}\right)$ be the associative algebra of formal pseudodifferential operators on $\left(S^{1}\right)^{n}$ (see $[\mathrm{A}]$ ). It is easy to check (see also $[\mathrm{A}]$ ) that the bracket of two such operators has coefficient zero at the term $x_{1}^{-1} \cdot \ldots \cdot x_{n}^{-1} \cdot \partial_{1}^{-1} \cdot \ldots \cdot \partial_{n}^{-1}$ (in any coordinate system). Therefore this coefficient defines a trace functional $\operatorname{Tr}: \Psi \operatorname{Dif}_{n}\left(S^{1}\right) \rightarrow \mathbb{C}$ which is known as a "noncommutative residue." Furthermore, $\operatorname{ad}\left(\ln x_{1}\right), \ldots$, $\operatorname{ad}\left(\ln x_{n}\right), \operatorname{ad}\left(\ln \partial_{1}\right), \ldots, \operatorname{ad}\left(\ln \partial_{n}\right)$ gives us a set of $2 n$ exterior derivations on the associative algebra $\Psi \operatorname{Dif}_{n}\left(S^{1}\right)$ satisfying conditions 1 ), (i) and (ii). It was shown in $[\mathrm{Sh}]$ that

$$
\begin{aligned}
& {[\ln \partial, \ln x]=} \\
& \quad \operatorname{ad}\left(x^{-1} \partial^{-1}+\frac{1}{2} x^{-2} \partial^{-2}+\frac{2}{3} x^{-3} \partial^{-3}+\ldots+\frac{(n-1) !}{n} x^{-n} \partial^{-n}+\ldots\right) .
\end{aligned}
$$

The $(2 n+1)$-cocycle constructed in [Sh] is not cohomologous to zero; it remains noncohomologous to zero when restricted to the Lie subalgebra Dif $_{n} \hookrightarrow$ $\Psi \operatorname{Dif}_{n}\left(S^{1}\right)$ of the (polynomial) differential operators on $\mathbb{C}^{n}$. The simplest way to check this fact is to construct its limit with respect to the deformation of the Lie algebra Poiss $2 n$ and to prove that this $(2 n+1)$-cocycle is not cohomologous to zero.

The characteristic feature of our situation is that the $2 n+3,2 n+5,2 n+7, \ldots$ - cocycles on the Lie algebra $\operatorname{Dif}_{n}$ constructed in this paper have quite complicated Hamiltonian limits; the question about their cohomological nontriviality remains open.

One final comment: it was proved in $[\mathrm{FT}]$ that

$$
H^{*}\left(\mathfrak{g l}_{\infty}^{\mathrm{fin}\left(\operatorname{Dif}_{n}\right)} ; \mathbb{C}\right)=\wedge^{*}\left(\xi_{2 n+1}, \xi_{2 n+3}, \xi_{2 n+5}, \ldots\right) ;
$$

furthermore, the trace on the associative algebra $\Psi \operatorname{Dif}_{n}\left(S^{1}\right)$ can be extended to the associative algebra $\mathfrak{g l}_{\infty}^{\mathrm{fin}\left(\Psi \operatorname{Dif}_{n}\left(S^{1}\right)\right)}$ and the derivations $\left\{\ln x_{i}, \ln \partial_{i}, i=\right.$ $1, \ldots, n\}$ also act on this algebra. This fact allows us to construct the corresponding cocycles on the Lie algebra $\mathfrak{g l}^{\mathrm{fin}_{\infty}\left(\mathrm{Dif}_{n}\right)}$, and our cocycles are pullbacks of these cocycles with respect to the inclusion $\operatorname{Dif}_{n} \hookrightarrow \mathfrak{g l}_{\infty}^{\mathrm{fin}\left(\operatorname{Dif}_{n}\right)}\left(D \mapsto D \cdot E_{11}\right)$. This construction together with the above result from $[\mathrm{FT}]$ imply that:

(1) Automorphisms of the Lie algebra $\Psi \operatorname{Dif}_{n}\left(S^{1}\right)$ act trivially on our cocycles;

(2) Another choice of the exterior derivations does not lead to new cohomological classes.

Actually, our methods allow us to construct just one unique cohomology class of the Lie algebra $\operatorname{Dif}_{n}$ in each odd dimension $\geq 2 n+1$.

I am grateful to B.L. Feigin for numerous valuable discussions. 


\section{Case $\left[D_{i}, D_{j}\right]=0$}

1.1. Let $\mathfrak{A}$ be an associative algebra with trace functional $\operatorname{Tr}: \mathfrak{A} \rightarrow \mathbb{C}$, and let $\left\{D_{1}, \ldots, D_{n}\right\}$ be a set of its (exterior) derivations satisfying the following condition:

$$
\operatorname{Tr}\left(D_{i} A\right)=0 \quad \text { for any } i=1, \ldots, n \text { and any } A \in \mathfrak{A} .
$$

Let $l \geq 1$ be an integer and let us consider the following expression:

$$
S_{a_{1}, \ldots, a_{n+2 l}}\left(A_{1}, \ldots, A_{n+2 l}\right)=\underset{A, D}{\operatorname{Alt}} \operatorname{Tr}\left(T_{1} \cdot \ldots \cdot T_{n+2 l}\right),
$$

where

(i) $A_{1}, \ldots, A_{n+2 l} \in \mathfrak{A}$;

(ii) $a_{1}, \ldots, a_{n+2 l} \in\{0,1\}$;

(iii) $T_{i}=D_{j(i)} A_{i}$ if $a_{i}=1 \quad\left(j(i)\right.$ is defined below) $T_{i}=A_{i}$ if $a_{i}=0$;

(iv) $a_{1}=1$ and $T_{1}=D_{1} A_{1}$;

(v) $j$ takes the values from 1 to $n$ in turn, i.e., if $i_{1}<i_{2}$ then $j\left(i_{1}\right)<j\left(i_{2}\right)$.

\section{Definition 1.}

$$
S_{\text {even }}=\sum_{\left(a_{1}, \ldots, a_{n+2 l}\right) \in a_{\text {even }}} S_{a_{1}, \ldots, a_{n+2 l}}\left(A_{1}, \ldots, A_{n+2 l}\right)
$$

where $a_{\text {even }}$ is the set of the sequences with values in $\{0,1\}$ of length $n+2 l$ and such that

(i) $a_{1}=1$;

(ii) $n$ of the $a_{i}$ 's are equal to 1 and $2 l$ of the $a_{i}$ 's are equal to 0 ;

(iii) if $a_{i}=1, a_{j}=1, j>i$ and $a_{i+1}, \ldots, a_{j-1}=0$, then $j-i$ is odd. In other words, there are an even number of 0 's between the two nearest 1's.

Remark 1. The condition (iii) should also hold for the "tail" of the sequence $\left(a_{j}\right)$, as if the numbers $a_{i}$ 's were placed on a circle.

Lemma 1. If $\left[D_{i}, D_{j}\right]=0$ for all $i, j=1, \ldots, n$ then $S_{\text {even }} \equiv 0$.

1.2. Proof of Lemma 1.1. Let us assign to each expression $S_{a_{1}, \ldots, a_{n+2 l}},\left(a_{i}\right) \in$ $a_{\text {even }}$ an expression $\widetilde{S}_{a_{1}, \ldots, a_{n+2 l}}\left(A_{1}, \ldots, A_{n+2 l}\right)$ in the following way:

$$
\widetilde{S}_{a_{1}, \ldots, a_{n+2 l}}=\sum_{j: a_{j}=1} \operatorname{Alt}_{A, D} \operatorname{Tr}\left(D_{j}\left(T_{1, j} \cdot \ldots \cdot T_{n+2 l, j}\right)\right)
$$

where $T_{i, j}=T_{i}$ for $i \neq j$ and $T_{j, j}=A_{j}$. Then Lemma 1.1 follows from the following statement:

Lemma 2. $\sum_{\left(a_{i}\right) \in a_{\text {even }}} \widetilde{S}_{a_{1}, \ldots, a_{n+2 l}}=(n+l) \cdot S_{\text {even }}$. 
Lemma 1.1 follows from this Lemma because of identity (2).

Proof of Lemma. Each summand in $\widetilde{S}_{a_{1}, \ldots, a_{n+2 l}}$ can be written as a further sum by the Leibniz rule. We have:

(6) $\underset{A, D}{\operatorname{Alt}} D_{j}\left(T_{1, j} \cdot \ldots \cdot T_{n+2 l, j}\right)=\sum_{i: T_{i, j}=A_{i}} \operatorname{Alt}_{A, D} \operatorname{Tr}\left(T_{1, j} \cdot \ldots \cdot D_{j} T_{i, j} \cdot \ldots \cdot T_{n+2 l, j}\right)$

because $\left[D_{i}, D_{j}\right]=0$ for all $i$. The summand in the r.h.s. of (6) corresponding to $i=j$ is equal to $S_{a_{1}, \ldots, a_{n+2 l}}$. As $j$ ranges from 1 to $n$ we obtain $n \cdot S_{a_{1}, \ldots, a_{n+2 l}}$. The other summands in the r.h.s. of (6) are of the following three types:

(i) Summands of the form $\operatorname{Alt}_{A, D} \operatorname{Tr}\left(T_{1, j} \cdot \ldots \cdot D_{j} T_{i, j} \cdot \ldots T_{n+2 l, j}\right) \quad\left(T_{i, j}=A_{i}\right)$ for which, when $i<j$, there exists $k$ such that $i<k<j$; when $i>j$, there exists $k$ such that $j<k<i$; and such that $a_{k}=1$.

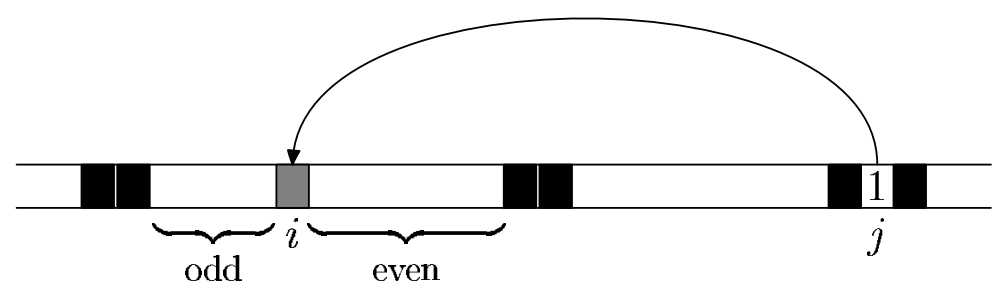

Figure 1

We move 1 from the $j$-th place to the $i$-th place, where it divides the sequence of zeroes of even length into two sequences. One of these sequences is necessarily of odd length, while the second sequence has even length. In Fig. 1, 1's are located on black squares before and after moving, and 0's are on white squares.

For each summand of this type there is a "dual" summand; the sum of a summand and its dual is 0. For example, the dual of Fig. 1 is Fig. 2 below:

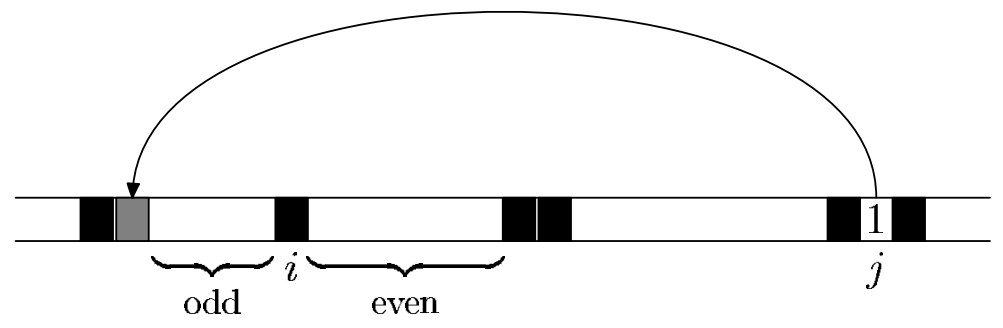

Figure 2

(ii) However, the dual summand for a summand of type (i) is not necessarily also of type (i). The typical situation is shown in Fig. 3 below.

Figure 3 correspons to the case when there does not exist $a_{k}=1$ between $a_{i}=0$ and where $a_{j}=0$ and $a_{j}$ divides the corresponding sequence into two 
odd parts. The two dual summands cancel one another because of the alternation of the $D$ 's.

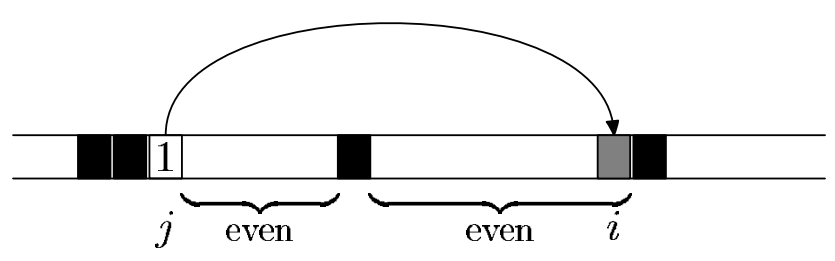

(ii)

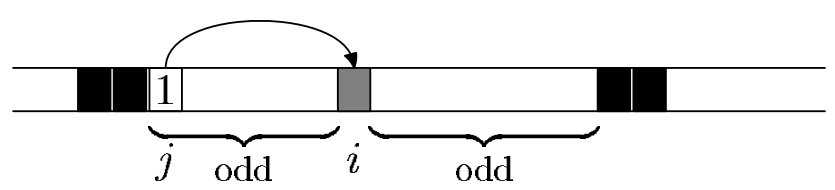

FiguRE 3. The summand of the type (i) and the dual summand of the type (ii)

(iii) There remain summands of a third type, which is shown schematically in Fig. 4. In this case there does not exist $a_{k}=1$ between $a_{i}=0$ and $a_{j}=0$, and $a_{j}$ divides the corresponding even sequence into two even parts. We differentiate types $(\mathrm{iii})_{1}$ and $(\mathrm{iii})_{2}$; strictly speaking, type (iii) $)_{1}$ is a special case of type $(\mathrm{iii})_{2}$.

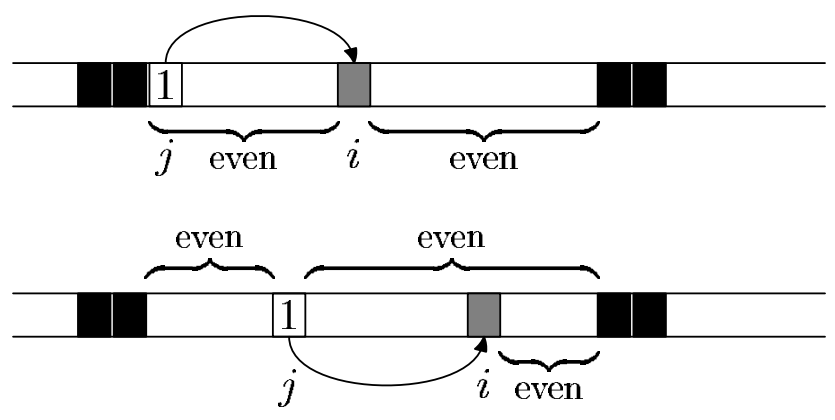

Figure 4. Types (iii) $)_{1}$ and (iii) $)_{2}$

Summands of type (iii) do not have dual summands, but they are of the form $S_{a_{1}, \ldots, a_{n+2 l}}$ for some $\left(a_{i}\right) \in a_{\text {even }}$, therefore they are summands of $S_{\text {even }}$. These summands together with the summands of the form $\operatorname{Alt}_{A, D} \operatorname{Tr}\left(T_{1, j} \cdot \ldots \cdot D_{j} T_{j, j} \cdot \ldots\right.$. $\left.T_{n+2 l, j}\right)$ lead to the coefficient $(n+l)$ in the statement of the Lemma.

1.3. Our aim is to construct the $(n+2 l-1)$-cocycle on the Lie algebra $\mathfrak{A}$ analogous to the $(n+1)$-cocycle given by formula (1).

Let us define the $(n+2 l-1)$-cochain $R_{a_{1}, \ldots, a_{n+2 l}}\left(A_{1}, \ldots, A_{n+2 l-1}\right)$ for each $S_{a_{1}, \ldots, a_{n+2 l}},\left(a_{i}\right) \in a_{\text {even }}$. Roughly speaking, we just shorten any sequence of consecutive zeroes to a single zero; after this procedure, the sequence will have 
odd length. We choose the first such sequence. More precisely, let us define the sequence $\left(\widetilde{a}_{i}\right)_{i=1, \ldots, n+2 l-1}$ for every sequence $\left(a_{i}\right) \in a_{\text {even }}$ in the following way:

Let $s_{1}=\min _{i}\left(a_{i}=0\right), s_{2}=\max _{i>s_{1}}\left(a_{i}=1\right)$; then:

- $\widetilde{a}_{1}, \ldots, \widetilde{a}_{s_{1}-1}=1$

- $\widetilde{a}_{s_{1}}, \ldots, \widetilde{a}_{s_{2}-2}=0$

- $\tilde{a}_{i}=a_{i+1}$ for $s_{2}-1 \leq i \leq n+2 l-1$.

Let $R_{a_{1}, \ldots, a_{n+2 l}}\left(A_{1}, \ldots, A_{n+2 l-1}\right)=\operatorname{Alt}_{A, D} \operatorname{Tr}\left(P_{1} \cdot \ldots \cdot P_{n+2 l-1}\right)$, where

- $P_{i}=D_{j(i)} A_{i}$ for $\widetilde{a}_{i}=1$,

- $P_{i}=A_{i}$ for $\widetilde{a}_{i}=0$

and $j(i)$ is defined in the following way: $j(1)=1 ; j\left(i_{1}\right)<j\left(i_{2}\right)$ when $i_{1}<i_{2}$ and $j=1, \ldots, n$. In other words, $j$ takes values from 1 to $n$ in turn.

\section{Lemma 3.}

$$
\begin{array}{r}
d\left(R_{a_{1}, \ldots, a_{n+2 l-1}}\right)\left(A_{n+2 l}, A_{1}, A_{2}, \ldots, A_{n+2 l-1}\right)= \\
(-1)^{n+2 l-s_{1}+1} \cdot S_{a_{1}, \ldots, a_{n+2 l}}\left(A_{1}, \ldots, A_{n+2 l}\right) .
\end{array}
$$

Proof. A direct calculation in the spirit of [Sh], Sections 1-3.

\section{Theorem 1.}

$$
\Psi_{n, l}^{0}=\sum_{\left(a_{i}\right) \in a_{\mathrm{even}}}(-1)^{s_{1}} R_{a_{1}, \ldots, a_{n+2 l}}\left(A_{1}, \ldots, A_{n+2 l-1}\right)
$$

is a $(n+2 l-1)$-cocycle on the Lie algebra $\mathfrak{A}$.

Proof. Follows from Lemma 1.1.

\section{Quantization}

2.1. Let $\mathfrak{A}$ be the associative algebra with trace $\operatorname{Tr} ; D_{1}, \ldots, D_{n}$ are its (exterior) derivations, which satisfy the following conditions:

$$
\begin{gathered}
{\left[D_{i}, D_{j}\right]=\operatorname{ad} Q_{i j} ; \quad Q_{i j}=-Q_{j i}} \\
\operatorname{Altt}_{i, j, k} D_{k}\left(Q_{i j}\right)=0
\end{gathered}
$$

In the case when $Q_{i j}=0$ for all $i, j$ we have constructed in Section 1 the $(n+2 l-1)$-cocycle on the Lie algebra $\mathfrak{A}$, for all integral $l \geq 1$. Our next problem is to quantize this cocycle and to find the $(n+2 l-1)$-cocycle for all $Q_{i j} \in \mathfrak{A}$ satisfying conditions (8) and (9).

In [Sh] such a cocycle was constructed for $l=1$ (quantization of formula (1)). Let us recall that construction.

Suppose that $n \geq 2$. We will consider intervals of length $n-2$ with some marked integral points, such that the distance between any two marked points greater or equal than 2 . Let us denote by $I_{k}$ the set of all such intervals with $k$ 
marked points $\left(1 \leq k \leq\left[\frac{n}{2}\right]\right)$. Denote by $1, \ldots, n-1$ the integral points of the interval.

Definition 2. Suppose that $t \in I_{k}$ and $i_{1}<\ldots<i_{k}$ are its marked points $\left(1 \leq i_{1}, i_{k} \leq\left[\frac{n}{2}\right]\right.$ and $i_{s+1}-i_{s} \geq 2$ for all $\left.s=1, \ldots, k-1\right)$. Then

$$
\mathcal{O}(t)=\underset{A, D}{\operatorname{Alt}} \operatorname{Tr}\left(P_{1, t} \cdot \ldots \cdot P_{n+1, t}\right)
$$

where

$$
\begin{aligned}
& \left.\begin{array}{rl}
P_{i, t} & =A_{j} \cdot Q_{j, j+1} \\
& =A_{j+1}
\end{array}\right\} \text { when } j=i_{s} \text { for some } s=1, \ldots, k \\
& P_{j+1, t}=A_{j+1} \quad \text { (i.e., if point } j \text { is marked) } \\
& P_{i, t}=D_{j} A_{j} \quad \text { when } j \text { and } j-1 \text { are not marked and } j \neq n+1 \\
& P_{n+1, t}=A_{n+1}
\end{aligned}
$$

Example 1. If $n=6$ and $t=\bullet-1 \bullet-1, t \in I_{2}$, then

$$
\mathcal{O}(t)=\operatorname{Alt}_{A, D} \operatorname{Tr}\left(A_{1} \cdot Q_{12} \cdot A_{2} \cdot D_{3} A_{3} \cdot A_{4} \cdot A_{5} \cdot D_{6} A_{6} \cdot A_{7}\right)
$$

If $t=\bullet \mapsto \bullet \mapsto, t \in I_{3}$, then

$$
\mathcal{O}(t)=\operatorname{Alt}_{A, D} \operatorname{Tr}\left(A_{1} \cdot Q_{12} \cdot A_{2} \cdot A_{3} \cdot Q_{34} \cdot A_{4} \cdot A_{5} \cdot Q_{56} \cdot A_{6} \cdot A_{7}\right)
$$

Remark 2. In formula (10) we are using the convention that $Q_{i j} "="\left[D_{i}, D_{j}\right]$ in the sense of the alternation on $D$. Furthermore, according to our convention we don't alternate the symbols $i$ and $j$ in $Q_{i j}$.

Theorem 2 (Main Conjecture from [Sh]). Let $\Sigma_{k}=\sum_{t \in I_{k}} \mathcal{O}(t)$. If conditions (8) and (9) hold, then

$$
\Psi_{n, 1}=\operatorname{Alt}_{A, D} \operatorname{Tr}\left(D_{1} A_{1} \cdot D_{2} A_{2} \cdot \ldots \cdot D_{n} A_{n} \cdot A_{n+1}\right)+\Sigma_{1}+\Sigma_{2}+\ldots+\Sigma_{\left[\frac{n}{2}\right]},
$$

is a $(n+1)$-cocycle on the Lie algebra $\mathfrak{A}$.

We will prove this Theorem in Section 3. 
2.2. Here we give a motivation for Theorem 2.1 and prove the Key Lemma, which we will use in Subsection 2.3 in order to formulate Theorem 2.3, which generalizes Theorem 2.1 to the case of arbitrary $l \geq 1$. We will prove this more general Theorem in Section 3.

Let us suppose that all derivations $D_{i}$ in the formula $(7)$ for a $(n+2 l-1)$ cocycle on the Lie algebra $\mathfrak{A}$ are inner, i.e., $D_{i} A=D_{i} \cdot A-A \cdot D_{i}, D_{i} \in \mathfrak{A}$; now we do not suppose that the condition $\left[D_{i}, D_{j}\right]=0$ hold. We define in this case the $(n+2 l-1)$-cochain $\Psi_{n, l}^{\text {inner }}$ by the formula (7). In the same way, we define the $(n+2 l)$-cochain $S_{\text {even }}^{\text {inner }}$ by the formulas (3), (4). It is clear that

$$
d \Psi_{n, l}^{\text {inner }}=S_{\text {even }}^{\text {inner }} .
$$

Now we replace all factors of the form $D_{i} A_{j}$ by $D_{i} \cdot A_{j}-A_{j} \cdot D_{i}$ in the formula and remove all parentheses. We obtain:

$$
\Psi_{n, l}^{\mathrm{inner}}=\widetilde{\Psi}_{n, l}^{\mathrm{inner}}+r_{n, l},
$$

where $\widetilde{\Psi}_{n, l}^{\mathrm{inner}}$ is the sum of all summands in $\Psi_{n, l}^{\mathrm{inner}}$ in which no two $D_{i}$ and $D_{j}$ are consecutive, and $r_{n, l}$ is the sum of the remaining summands in $\Psi_{n, l}^{\text {inner }}$, i.e., those of the form $\ldots \cdot D_{i} \cdot D_{j} \cdot \ldots$ for some $i, j$.

Key Lemma 1. $\widetilde{\Psi}_{n, l}^{\text {inner }}$ is a $(n+2 l-1)$-cocycle on the Lie algebra $\mathfrak{A}$.

Remark 3. In fact, $\widetilde{\Psi}_{n, l}^{\text {inner }}$ is a coboundary.

Proof of Key Lemma. This Lemma is similar to Lemma 1.1, however, formally it is another statement. By analogy with (13), we have a similar decomposition of $S_{\text {even }}^{\text {inner }}$ :

$$
S_{\text {even }}^{\text {inner }}=\widetilde{S}_{\text {even }}^{\text {inner }}+r_{\text {even }}
$$

\section{Lemma 4.}

(i) $d \widetilde{\Psi}_{n, l}^{\text {inner }}=\widetilde{S}_{\text {even }}^{\text {inner }}$;

(ii) $d r_{n, l}=r_{\text {even }}$.

Proof. Follows from formula (12).

Now all that remains is to prove that $\widetilde{S}_{\text {even }}^{\text {inner }} \equiv 0$ (without any conditions about the inner derivations $D_{i}$ ). This is a direct calculation in the spirit of Lemma 1.1 (see Lemma 4.2 of [Sh] for the case $l=1$ ).

Furthermore, in the case when all $D_{i}$ are inner derivations satisfying condition (8), $r_{n, l}$ is represented by the sum $\Sigma$ of the terms of the form $\mathcal{O}(t)$, where $t$ is an interval with marked points (see (10)). We increase $D_{i} \cdot D_{j}$ by $\left[D_{i}, D_{j}\right]$ via the alternation. Hence $\Psi_{n, l}^{\text {inner }}-\Sigma$ is a cocycle (which, in fact, is cohomologous to zero). If now the $D_{i}$ 's are arbitrary derivations (not necessary inner) satisfying condition (8), then it turns out that this expression continues to be a cocycle, whenever condition (9) holds. This was proved in [Sh] in the case $l=1$ and 
$n=4$, in the first nontrivial case. In the case $l=1$ this reasoning leads to the formulation of Theorem 2.1.

2.3. Case of arbitrary $l \geq 1$. We have:

$$
\widetilde{\Psi}_{n, l}^{\text {inner }}=\sum_{\left(a_{i}\right) \in a_{\text {even }}}(-1)^{s_{1}} \cdot \widetilde{R}_{a_{1}, \ldots, a_{n+2 l}}\left(A_{1}, \ldots, A_{n+2 l-1}\right)
$$

where:

$$
\widetilde{R}_{a_{1}, \ldots, a_{n+2 l}}=\widetilde{\operatorname{Alt}} \operatorname{Tr}\left(P_{1} \cdot \ldots \cdot P_{n+2 l}\right)
$$

and

- $P_{i}=\left[D_{j(i)}, A_{i}\right]$ when $\widetilde{a}_{i}=1$;

- $P_{i}=A_{i}$ when $\widetilde{a}_{i}=0$;

- $j(1)=1$ and the indices $j$ takes the values from 1 to $n$ in term (formula (7)).

The sign " " in the r.h.s. of (16) means the sum on all the terms, in which no two $D_{i}, D_{j}$ are consecutive.

We quantize each summand $R_{a_{1}, \ldots, a_{n+2 l}}$ separately.

\section{Definition 3.}

(i) Denote by $\operatorname{Circle}_{k}^{a_{1}, \ldots, a_{n+2 l}}$ the set of all circles with $n+2 l-1$ integral points from which $k\left(1 \leq k \leq\left[\frac{n}{2}\right]\right)$ are marked. The distance between any two marked points is $\geq 2$. The points are enumerated by $1, \ldots, n+$ $2 l-1$. Point $i$ may be marked only if $\widetilde{a}_{i}=1$ and $\widetilde{a}_{i+1}=1$ (we suppose that $\left.\widetilde{a}_{n+2 l}=\widetilde{a}_{1}\right)$.

(ii) For $t \in \operatorname{Circle}_{k}^{a_{1}, \ldots, a_{n+2 l}}$ we define $\mathcal{O}(t)$ by analogy with Definition 2.1 after replacing the interval with the circle.

(iii) $\Sigma_{k}^{a_{1}, \ldots, a_{n+2 l}}=\sum_{t \in \text { Circle }_{k}^{a_{1}, \ldots, a_{n+2 l}}} \mathcal{O}(t)$.

Theorem 3. Let $\mathfrak{A}$ be an associative algebra with $\mathrm{Tr}$, and let $D_{1}, \ldots, D_{n}$ be its (exterior) derivations, which satisfy conditions (8), and (9). Then

$$
\Psi_{n, l}=\Psi_{n, l}^{0}+\sum_{\left(a_{i}\right) \in a_{\text {even }}}(-1)^{s_{1}} \sum_{k \geq 1} \Sigma_{k}^{a_{1}, \ldots, a_{n+2 l}}
$$

is a $(n+2 l-1)$-cocycle on the Lie algebra $\mathfrak{A}$.

\section{Proofs}

We prove here Theorem 2.1 and Theorem 2.3. Actually we prove Theorem 2.1 only (the case $l=1$ ) but one can easily generalize the proof to the case of an arbitrary integer $l \geq 1$ (Theorem 2.3). 
3.1. Let $\mathfrak{A}$ be an associative algebra with trace functional $\mathrm{Tr}$, and let $D_{1}, \ldots, D_{n}$ be its derivations, which satisfy conditions (8) and (9).

According to Lemma 1.1, we have:

$$
\begin{aligned}
& \underset{A, D}{\operatorname{Alt}} \operatorname{Tr}\left\{D_{1}\left(A_{1} \cdot D_{2} A_{2} \cdot \ldots \cdot D_{n} A_{n} \cdot A_{n+1} \cdot A_{n+2}\right)\right. \\
&+D_{2}\left(D_{1} A_{1} \cdot A_{2} \cdot D_{3} A_{3} \cdot \ldots \cdot D_{n} A_{n} \cdot A_{n+1} \cdot A_{n+2}\right) \\
&+\ldots \ldots \ldots \ldots \ldots \ldots \ldots \ldots \ldots \ldots \ldots \ldots \ldots \ldots \ldots \ldots \ldots \ldots \ldots \ldots \ldots \\
&\left.+D_{n}\left(D_{1} A_{1} \cdot \ldots \cdot D_{n-1} A_{n-1} \cdot A_{n} \cdot A_{n+1} \cdot A_{n+2}\right)\right\} \\
& \quad=(n+1) \cdot \operatorname{Alt}_{A, D} \operatorname{Tr}\left(D_{1} A_{1} \cdot \ldots \cdot D_{n} A_{n} \cdot A_{n+1} \cdot A_{n+2}\right)+\Phi_{1},
\end{aligned}
$$

where $\Phi_{1}$ consists of terms linear on $Q_{i j}$. Let us denote the l.h.s. of (17) by $X_{1}$. Our aim is to represent $\Phi_{1}$ modulo the trace of the full derivations as a sum of terms of the form $d(\mathcal{O}(t)$ ) (where $t$ is an interval of length $2 n-2$ with 1 marked point(see Subsection 2.1) and $d$ is the differential in the cochain complex of the Lie algebra $\mathfrak{A})$ and the expression $\Phi_{2}$, consisting of terms quadratic in $Q_{i j}$, and so on.

We have:

$$
\begin{aligned}
& \text { (18) } \Phi_{1}=\underset{A, D}{\operatorname{Alt}} \operatorname{Tr}\left\{A_{1} \cdot Q_{12} \cdot A_{2} \cdot D_{3} A_{3} \cdot \ldots-A_{1} \cdot A_{2} \cdot Q_{12} \cdot D_{3} A_{3} \cdot \ldots\right. \\
& \left.+A_{1} \cdot D_{2} A_{2} \cdot Q_{13} \cdot A_{3} \cdot D_{4} A_{4} \cdot \ldots-A_{1} \cdot D_{2} A_{2} \cdot A_{3} \cdot Q_{13} \cdot D_{4} A_{4} \cdot \ldots+\ldots-\ldots\right\}
\end{aligned}
$$

Now suppose that

$$
\begin{gathered}
X_{2}=\underset{A, D}{\operatorname{Alt}} \operatorname{Tr}\left\{-D_{3}\left(A_{1} \cdot A_{2} \cdot Q_{12} \cdot A_{3} \cdot D_{4} A_{4} \cdot \ldots\right)+D_{2}\left(A_{1} \cdot A_{2} \cdot Q_{13} \cdot A_{3} \cdot D_{4} A_{4} \cdot \ldots\right)\right. \\
\left.-D_{4}\left(A_{1} \cdot D_{2} A_{2} \cdot A_{3} \cdot Q_{13} \cdot A_{4} \cdot D_{5} A_{5} \cdot \ldots\right)+\ldots-\ldots\right\} .
\end{gathered}
$$

The first, second, and third summands in (19) correspond to the second, third, and fourth summands in (18), respectively.

In general, the summands of (18) are of the following two types:

(i) summands which contain products of the form $A \cdot Q \cdot A$ - there are no summands in (19) corresponding to the summands of (18) of this type;

(ii) the remaining summands in (18) have the form

$$
\ldots \cdot D_{i} A_{i-1} \cdot D_{i} A_{i} \cdot Q_{j, i+1} \cdot A_{i+1} \cdot D_{i+2} A_{i+2} \cdot \ldots
$$

or

$$
\ldots \cdot D_{i-1} A_{i-1} \cdot A_{i} \cdot Q_{j, i} \cdot D_{i+1} A_{i+1} \cdot \ldots ;
$$

the following summands of (19)

$$
D_{i}\left(\ldots \cdot D_{i-1} A_{i-1} \cdot A_{i} \cdot Q_{j, i+1} \cdot A_{i+1} \cdot D_{i+2} A_{i+2} \cdot \ldots\right)
$$

and

$$
D_{i+1}\left(\ldots \cdot D_{i-1} A_{i-1} \cdot A_{i} \cdot Q_{j, i} \cdot A_{i+1} \cdot D_{i+2} A_{i+2} \cdot \ldots\right)
$$

correspond to the above two summands of (18), respectively. 


\section{Lemma 5.}

$$
X_{2}=2 \Phi_{1}-d\left((2 n+2) \cdot \sum_{t \in I_{1}} \mathcal{O}(t)\right)-\Phi_{2},
$$

where $\Phi_{2}$ consists of expressions quadratic in $Q_{i j}$ and $d$ is the differential in the cochain complex of the Lie algebra $\mathfrak{A}$.

Proof. A straightforward calculation.

In the sequel we will need an explicit form for the summands of $\Phi_{2}$ : they may be of the following two types:

(i) $\ldots \cdot D A \cdot \underline{A} \cdot D A \cdot \ldots \cdot D A \cdot A \cdot Q \cdot A \cdot D A \cdot \ldots \cdot D A \cdot A \cdot Q \cdot D A \cdot \ldots$

(ii) $\ldots \cdot D A \cdot \underline{A} \cdot D A \cdot \ldots \cdot D A \cdot \underline{A \cdot Q \cdot A} \cdot D A \cdot \ldots \cdot D A \cdot \underline{Q \cdot A} \cdot D A \cdot \ldots$

3.2. Starting from $\Phi_{2}$ we construct an expression $X_{3}$ in the following way: there are two different cases for expressions given by the formulas (21):

1) expressions which contain two factors of the form $A \cdot Q \cdot A$. They have the following form:

$$
\ldots \cdot A \cdot Q \cdot A \cdot \ldots \cdot A \cdot Q \cdot A \cdot \ldots ;
$$

there do not exist any corresponding summands in $X_{3}$;

2) to

$\xi=\ldots D A \cdot \underline{A_{i}} \cdot D A \cdot \ldots \cdot D A \cdot \underline{A \cdot Q} \cdot \underline{A} \cdot D A \cdot \ldots \cdot D A \cdot A_{k} \cdot Q_{j, k} \cdot D_{k+1} A_{k+1} \cdot \ldots$

corresponds an expression

$$
\widetilde{\xi}=D_{k+1}\left(\ldots \cdot D A \cdot \underline{A_{i}} \cdot D A \cdot \ldots \cdot D A \cdot \underline{A_{k} \cdot Q_{j, k} \cdot A_{k+1}} \cdot D A \cdot \ldots\right)
$$

and to

$\xi=D A \cdot \underline{A_{i}} \cdot D A \cdot \ldots \cdot D A \cdot \underline{A \cdot Q \cdot A} \cdot D A \cdot \ldots \cdot D A \cdot D_{k-1} A_{k-1} \cdot \underline{Q_{j k}} \cdot A_{k} \cdot D A \cdot \ldots$

corresponds an expression

$$
\widetilde{\xi}=D_{k-1}\left(\ldots \cdot D A \cdot \underline{A_{i}} \cdot D A \cdot \ldots \cdot D A \cdot \underline{A_{k-1} \cdot Q_{j, k} \cdot A_{k}} \cdot \ldots\right)
$$

\section{Definition 4.}

$$
X_{3}=\sum_{\substack{\text { all summands of } \Phi_{2} \\ \text { of type 2 }}} \operatorname{Alt}_{A, D} \operatorname{Tr} \widetilde{\xi}
$$

\section{Lemma 6.}

$$
X_{3}=2 \Phi_{2}-d\left((2 n+2) \sum_{t \in I_{2}} \mathcal{O}(t)\right)-\Phi_{3}
$$

where $\Phi_{3}$ is a sum of expressions cubic in $Q_{i j}$. 
3.3. Proof of Theorem 2.1. We define the sequence $X_{1}, X_{2}, X_{3}, \ldots$ by formulas analogous to (20) and (22) and prove Lemmas analogous to Lemma 3.1 and 3.2. All that remains is to note that every $X_{i} \equiv 0$ by formula (2).

\section{Conjecture 1.}

$$
H^{*}\left(\mathfrak{g l}_{\infty}^{\mathrm{fin}\left(\operatorname{Dif}_{n}\right)} ; \mathbb{C}\right)=\wedge^{*}\left(\Psi_{n, 1}, \Psi_{n, 2}, \Psi_{n, 3}, \ldots\right)
$$

\section{References}

[A] M. Adler. On a trace functional for formal pseudo-differential operators and the symplectic structure of the Korteweg - de Vries type equations, Invent. Math. 50 (1979) 219-248.

[FT] B.L. Feigin and B.L. Tsygan. Cohomology of the Lie algebra of the generalized Jacobian matrices (Russian), Funktsional. Anal. i Prilozhen. 17 (1983), 86-87.

[KK] O.S Kravchenko and B.A. Khesin. Central extensions of the algebra of pseudo-differential symbols (Russian), Funktsional. Anal. i Prilozhen. 25 (1991), 83-85, translation in Functional Anal. Appl. 25 (1991), 152-154.

[Sh] Shoikhet B. Cohomology of the Lie algebras of differential operators : lifting formulas, q--alg/9712007.

IUM, 11 Bol'shoj Vlas'evskij Per., Moscow 121002, Russia

E-mail address: borya@mccme.ru 